TecnoLógicas

ISSN-p 0123-7799

ISSN-e 2256-5337

Vol. 23, No. 48, pp. 17-29

Mayo-agosto de 2020

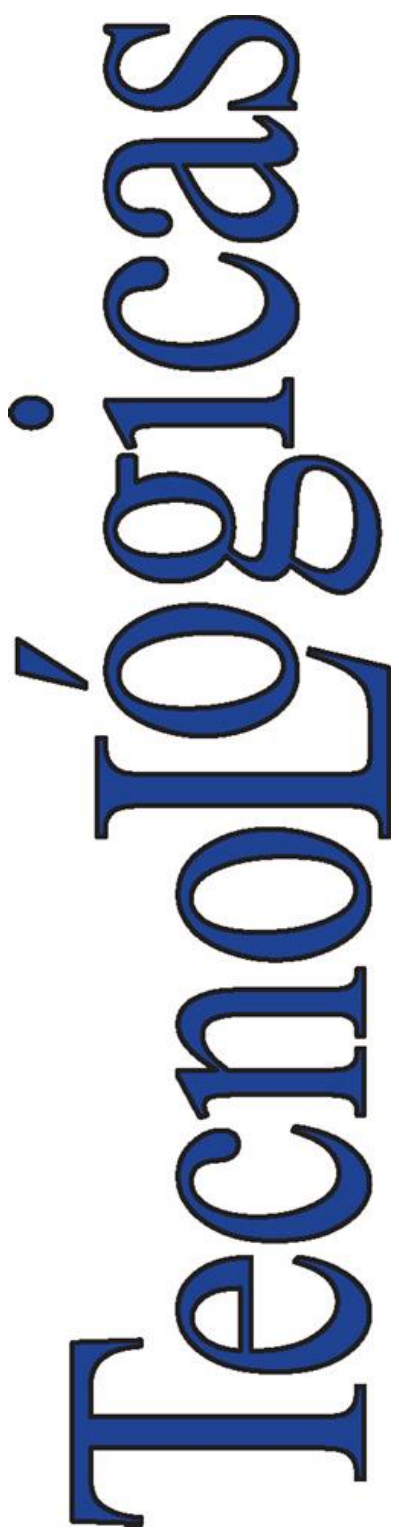

(C) Instituto Tecnológico Metropolitano Este trabajo está licenciado bajo una Licencia Internacional Creative Commons Atribución (CC BY-NC-SA)

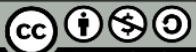

Artículo de Investigación/Research Article

\section{Potencial agroindustrial del epicarpio de mandarina como alternativa de colorante natural en pan}

\section{Agro-Industrial Potential of the Mandarina Epicarp a Natural Coloring Allternative in Bread}

\author{
Luis E. Ordoñez-Santos (D) ${ }^{1}$, \\ Jessica Esparza-Estrada $\mathbf{Q}^{2}, \mathrm{y}$ \\ Pedro Vanegas-Mahecha (D) 3
}

Recibido: 10 de agosto de 2019

Aceptado: 12 de diciembre de 2019

Cómo citar / How to cite

L. E. Ordoñez-Santos, J. Esparza-Estrada, P. Vanegas-Mahecha, "Potencial agroindustrial del epicarpio de mandarina como alternativa de colorante natural en pan”, TecnoLógicas, vol. 23, no. 48, pp. 17 29, 2020. https://doi.org/10.22430/22565337.1465

PhD, en Ciencias Biológicas, Facultad de Ingeniería y Administración, Departamento de Ingeniería, Universidad Nacional de Colombia, PalmiraColombia, leordonezs@unal.edu.co

2 Ingeniera Agroindustrial, Facultad de Ingeniería y Administración, Departamento de Ingeniería, Universidad Nacional de Colombia, PalmiraColombia, jesparzae@unal.edu.co

3 MSc. en Ingeniería Agrícola, Facultad de Ingeniería y Administración, Departamento de Ingeniería, Universidad Nacional de Colombia, PalmiraColombia, pvanegasmah@unal.edu.co 


\section{Resumen}

En este estudio se evaluó el potencial agroindustrial de la harina de epicarpio de mandarina Oneco (Citrus reticulata) como posible colorante natural en el pan. En la metodología se analizaron las propiedades fisicoquímicas como el $\mathrm{pH}$, aw, los parámetros de color: luminosidad $\left(\mathrm{L}^{*}\right)$, cromaticidad $\left(\mathrm{C}^{*}\right)$ y ángulo de tono $\left(\mathrm{h}^{\circ}\right)$, porcentaje de acidez, porcentaje de materia seca, cuantificación de carotenoides por espectrofotometría, compuestos fenólicos, capacidad antioxidante en harina y parámetros de color $\left(\mathrm{L}^{*}, \mathrm{C}^{*} \mathrm{y} \mathrm{h} \mathrm{h}^{\circ}\right)$ en el pan. Se encontró que, después del tratamiento de secado convectivo, se retuvieron concentraciones significativamente más altas de compuestos bioactivos como los fenólicos, se evidenció también un aumento en la capacidad antioxidante y el contenido de carotenoides, además de cambios significativos en los parámetros de color $\left(\mathrm{L}^{*}\right.$ y $\left.\mathrm{C}^{*}\right)$. En la sustitución de la harina de epicarpio de mandarina en las formulaciones del pan, se notó un aumento significativo en la concentración del contenido de carotenoides, siendo el pico de esta en la sustitución del $4 \%$; En los parámetros de color, se hallaron diferencias significativas en los parámetros $\mathrm{L}^{*}$ y $\mathrm{C}^{*}$, presentando el mayor valor de $\mathrm{L}^{*}$ en la sustitución del $2 \%$ y de $\mathrm{C}^{*}$ en la sustitución del $4 \%$, mientras que el parámetro $\mathrm{h}^{\circ}$ no presentó diferencias significativas en ninguna de las formulaciones. Finalmente, se concluye que la harina de epicarpio de mandarina puede ser una alternativa importante, como aditivo de colorante natural en la elaboración del pan.

\section{Palabras clave}

Carotenoides, compuestos fenólicos, capacidad antioxidante, cítrico, cromaticidad.

\section{Abstract}

This study evaluated the agro-industrial potential of flour made of the epicarp of Oneco mandarin (Citrus reticulata) as a possible natural bread coloring. We analyzed the physiochemical properties of said fruit, such as $\mathrm{pH}$, aw, color parameters (luminosity, $\mathrm{L}^{*}$; chromaticity, $\mathrm{C}^{*}$; and pitch angle, $\mathrm{h}^{\circ}$ ), acidity percentage, dry matter percentage, quantification of carotenoids by spectrophotometry, phenolic compounds, antioxidant capacity in flour, and color parameters $\left(\mathrm{L}^{*}, \mathrm{C}^{*}\right.$, and $\left.\mathrm{h}^{\circ}\right)$ in bread. We found that, after convective drying, the fruit retained significantly higher concentrations of bioactive compounds, such as phenols. We also observed an increase in antioxidant capacity and carotenoid content, in addition to significant changes in the color parameters $\left(\mathrm{L}^{*}\right.$ and $\left.\mathrm{C}^{*}\right)$. When the mandarin epicarp flour substituted conventional flour in the bread formula, we noted a significant increase in the concentration of the carotenoid content; the peak of this substitution was 4\%. Regarding color, parameters $\mathrm{L}^{*}$ and $\mathrm{C}^{*}$ exhibited significant differences; $L^{*}$ was higher in the $2 \%$ substitution, while $\mathrm{C}^{*}$ was higher in the $4 \%$ substitution, and $\mathrm{h}^{\circ}$ did not present significant differences in any of the formulas. Finally, we conclude that mandarin epicarp flour can be an important alternative additive for natural coloring in bread making.

\section{Keywords}

Carotenoids, Phenolic compounds, Antioxidant capacity, Citric, Chromaticity. 


\section{INTRODUCCIÓN}

Los cítricos son uno de los cultivos frutales más importantes del mundo, con una producción anual de aproximadamente 102 millones de toneladas [1]. Estas frutas son originarias de las zonas tropicales y subtropicales del Sureste Asiático, el noreste de la India, el Sur de China, y el Archipiélago Malayo [2]. La mandarina es una especie subtropical de clima templado perteneciente a la familia Rutaceae y al género Citrus L., es un fruto globoso, de color anaranjado uniforme, sabor dulce, corteza granulada y de tamaño mediano [3].

El procesamiento de los cítricos genera subproductos que pueden alcanzar hasta el $50 \%$ del peso original de la fruta, siendo el epicarpio el subproducto principal [4], [5].

Estos subproductos son fuentes importantes de aceite esencial, carotenoides y compuestos fenólicos, que los convierte en recursos atractivos en la valoración agroindustrial como fuente importante de aditivos en la industria alimentaria, cosmética y farmacéutica [6], [7]. Estudios previos reconocen a los cítricos como frutas que promueven la salud, debido a la presencia de estos compuestos bioactivos [8]. Las dietas ricas en fitoquímicos como los carotenoides y los compuestos fenólicos se han asociado con la reducción de enfermedades como ciertos tipos de cáncer, inflamación, problemas cardiovasculares, cataratas, degeneración macular y enfermedades neurodegenerativas [9]. Los carotenoides presentes en los cítricos amarillos y verdes, están ganando importancia en los productos alimenticios, debido a sus beneficios relacionados a la salud (ricos en provitamina A y efecto anticancerígeno) [10].

Se han realizado varios estudios sobre la caracterización de frutas cítricas, como los llevados a cabo por [4], los autores caracterizaron cuatro especies de Citrus cultivadas en Brasil (C. sinensis, cvs. Pera y Lima; C. latifolia tanaka cv. Tahiti; $C$. limettioides tanaka $c v$. Sweet lime y $C$. reticulate, $c v$. Ponkan) en relación con el contenido de minerales, ácido ascórbico, polifenoles totales y capacidad antioxidante de pulpas y epicarpio. Los autores encontraron que la mandarina Ponkan presentó mayor capacidad antioxidante y, en general, el epicarpio de las variedades estudiadas presentó un contenido estadísticamente mayor de todos los compuestos evaluados en comparación de la pulpa; [10] determinó el contenido total de carotenoides, vitamina C y capacidad antioxidante de tres variedades de cítricos cultivados en la parte sur de Corea, tales son Yuza (Citrus junos sieb ex tabaka), Kjool (Citrus unshiu marcow) y Dangyooja (Citrus grandisosbeck). Los autores identificaron siete carotenoides de los cuales predominan la B-criptoxantina, astaxantina y zeaxantina, además, encontraron que la variedad Yuza contiene cantidades más altas de carotenoides y vitamina C; [11] estimó los polifenoles y flavonoides presentes en la pulpa $\mathrm{y}$ epicarpio de limón de Bhopal e India, también determinó el poder reductor, la actividad antioxidante y la inhibición del peróxido de lípidos. Los autores encontraron que el epicarpio de limón presentó valores estadísticamente mayores de los análisis realizados en comparación con la pulpa; [12] estudiaron el perfil fisicoquímico y fitoquímico, así como las propiedades antioxidantes en cítricos como la naranja 'Valencia' (Citrus sinensis, cv.), pomelo (Citrus paradisi, cus. 'White Marsh', 'Star Ruby', 'Rio Red') y un híbrido 'Mandora' (Citrus reticulata $x$ Citrus sinensis, $c v$ ). Los autores hallaron que la fruta de 'Valencia' presentó un mayor contenido de ácido fenólico, flavonoide y ascórbico, así como una mayor capacidad antioxidante.

Se han adelantado varios estudios sobre el uso de harinas de epicarpio y mesocarpio de frutas en productos horneados. [13] evaluaron los parámetros fisicoquímicos $\mathrm{y}$ 
sensoriales del uso de harina de epicarpio de chontaduro en torta dulce. [14] determinó las propiedades fisicoquímicas y morfológicas y la viscosidad de la harina de castaña de agua india en la preparación de galletas. [15] caracterizó la harina de epicarpio de naranja y maracuyá y su uso en pan y torta dulce. [16] evaluó las propiedades reológicas, químicas $\mathrm{y}$ sensoriales y el perfil de textura del uso de harina de granos de café en galletas.

En la actualidad son limitados los estudios en la valoración de los subproductos generados en el procesamiento de críticos en Colombia que permitan identificar el potencial agroindustrial del epicarpio de mandarina como fuente de aditivos naturales en la industria alimentaria, cosmética $y$ farmacéutica. En razón a esto, el objetivo de la presente investigación fue evaluar el potencial agroindustrial de la harina de epicarpio de mandarina Oneco (Citrus reticulata) como posible colorante natural en el pan.

\section{MATERIALES Y MÉTODOS}

\subsection{Acondicionamiento de material primo}

De un supermercado ubicado en la galería Alameda en la ciudad de Santiago de Cali (Valle del Cauca), se adquirieron 20 $\mathrm{kg}$ de mandarina Oneco (Citrus reticulata) en estado de madurez de consumo, de los cuales se obtuvieron $4 \mathrm{~kg}$ de epicarpio aproximadamente. La mandarina fue lavada en una solución jabonosa 1:4, desinfectada con hipoclorito a 100ppm durante 5 min y pelada manualmente. El epicarpio (cáscara) de la mandarina fue troceado en julianas de $2,8 \mathrm{~mm}$ de espesor y $6,5 \mathrm{~cm}$ de longitud aproximadamente, sumergido en una solución de metasulfito a $100 \mathrm{ppm}$ durante $5 \mathrm{~min}$, escaldado durante 2 min a $85{ }^{\circ} \mathrm{C}$ y congelado con el fin de reducir el tiempo de secado. El epicarpio fue tratado durante 4 horas aproximadamente en un secador de bandejas convectivo a $40{ }^{\circ} \mathrm{C}$ y velocidad de flujo a $3 \mathrm{~m} / \mathrm{s}$, hasta obtener una humedad constante de $8 \%$. Se redujo el tamaño del epicarpio deshidratado en un molino de cuchillas IKA Labortchnik M hasta que el $98 \%$ de la harina pasó por una malla US 200. Posteriormente, la harina de epicarpio de mandarina fue almacenada en un frasco de vidrio bajo refrigeración a una temperatura de $4{ }^{\circ} \mathrm{C}$ [17].

\subsection{Aplicación de la harina de epicarpio de mandarina en el pan}

El pan se elaboró siguiendo la metodología de [18] con algunas modificaciones; los ingredientes fueron: 150 $\mathrm{g}$ de harina de trigo, $54 \mathrm{~g}$ de agua, $37 \mathrm{~g}$ de margarina, $15 \mathrm{~g}$ de azúcar, $15 \mathrm{~g}$ de huevos, $3 \mathrm{~g}$ de sal y $3 \mathrm{~g}$ de levadura fresca; los ingredientes fueron amasados de forma manual durante $10 \mathrm{~min}$, pasado este tiempo se dejó reposar la masa durante 15 min a temperatura ambiente, posteriormente se dividió la masa en porciones de 22 g y se leudó a $32{ }^{\circ} \mathrm{C}$ y $54 \%$ humedad durante $40 \mathrm{~min}$ hasta triplicar su tamaño. Por último, se horneó la masa en un horno Óster a $180^{\circ} \mathrm{C}$ durante $20 \mathrm{~min}$.

Se realizaron cuatro tratamientos, el primer tratamiento fue el control con 0,03 $\mathrm{g}$ de tartrazina y los otros tres tratamientos fueron sustituciones de harina de trigo por harina de epicarpio de mandarina a $2 \%, 3 \%$ y $4 \%$.

\subsection{Determinaciones fisicoquímicas}

A las muestras de estudio se le hicieron mediciones de materia seca siguiendo el protocolo [19], en una balanza de humedad Precisa XM60, e= 0,01, Suiza; de actividad de agua siguiendo el protocolo [19] en el equipo AQUALAB, 4TE, México; de $\mathrm{pH}$ siguiendo el protocolo [20], en el pH-metro SI Analytics, Lab 855; de porcentaje de acidez referido a ácido cítrico siguiendo el protocolo [21]. 


\subsection{Determinación del color superficial CIEL* $\mathbf{a}^{*} \mathbf{b}^{*}$}

Se les determinó el color a las muestras de estudio mediante la lectura de las coordenadas $\mathrm{L}^{*}, \mathrm{a}^{*} \mathrm{y}$ b* en un colorímetro Konica Minolta CR-400. El colorímetro se estandarizó con una baldosa negra y una blanca $(Y=89,5 ; \mathrm{x}=0,3176 ; \mathrm{y} y=0,3347)$ usando el iluminante D65 y un observador $2^{\circ}$. Los valores numéricos de $L^{*}, a^{*} y b^{*}$ se convirtieron en croma $\left(\mathrm{C}^{*}\right)$, y ángulo de matiz $\left(h^{\circ}\right)$ según [22], mediante (1) y (2):

$$
\begin{aligned}
& C^{*}=\left(a^{*^{2}}+b^{*^{2}}\right)^{\frac{1}{2}} \\
& h^{\mathrm{o}}=\tan ^{-1}\left(b^{*} / a^{*}\right)
\end{aligned}
$$

\subsection{Determinación del contenido de carotenoides}

Los carotenoides se cuantificaron siguiendo la metodología de [22]. Se pesaron $0,1 \mathrm{~g}$ de muestra triturada en un tubo de ensayo, se adicionaron $7 \mathrm{ml}$ de una solución de etanol/hexano a una relación 4:3. Los tubos de ensayo se pusieron en un matraz con agua helada y se cubrieron con una bolsa negra para protegerlos de la luz.

Posteriormente, se sometieron a agitación a $140 \mathrm{rpm}$ en una plancha de agitación durante $1 \mathrm{~h}$. Transcurrido el tiempo se adicionó $1 \mathrm{ml}$ de agua destilada y se sometieron a agitación durante $10 \mathrm{~min}$ más.

Se visualizó una separación de fases; en la parte superior el hexano y en la parte inferior el etanol y agua. Se leyó en el espectrofotómetro Spectrophotometer Jenway 6320D, USA la fase orgánica (hexano) a $450,444,451$ y $472 \mathrm{~nm}$ para Bcaroteno, a-caroteno, B-criptoxantina, zeaxantina y licopeno respectivamente. El contenido de carotenoides disueltos en hexano se calculó mediante (3):

$$
\frac{m g \text { carotenoide }}{100 g}=\frac{A * V * 10^{4}}{A^{1 \%} l c m * P}
$$

Donde A es la absorbancia, V el volumen de la capa de hexano, $\mathrm{P}$ es el peso de la muestra (g), y $A^{1 \%} l \mathrm{~cm}$ es el coeficiente de extinción: 2560, 2800, 2460, 2480 y 3540 para B-caroteno, a-caroteno, Bcriptoxantina, zeaxantina y licopeno respectivamente.

\subsection{Determinación de compuestos fenólicos totales}

La concentración de fenoles totales se determinó siguiendo la metodología de [22]. Se pesaron $0,25 \mathrm{~g}$ de muestra en un tubo de ensayo forrado con papel aluminio y se adicionaron $10 \mathrm{ml}$ de etanol-agua a una relación de 80:20\%v/v. Se agitó en una plancha de agitación a $150 \mathrm{rpm}$ durante tres minutos y posteriormente se sonificó la mezcla durante 20 minutos a $25^{\circ} \mathrm{C}$.

Pasado este tiempo se filtró el extracto y se tomaron y mezclaron $0,5 \mathrm{ml}$ del extracto con $5 \mathrm{ml}$ de agua destilada y 0,5 $\mathrm{ml}$ de reactivo de Folin-Ciocalteu; entonces, se dejó que la mezcla reaccionara durante 2 min a temperatura ambiente.

Pasado el tiempo, se adicionó $1 \mathrm{ml}$ de carbonato de sodio anhídrido al $20 \%$ y se sometieron los tubos a baño térmico durante $15 \min$ a $45^{\circ} \mathrm{C}$. Se dejó enfriar a temperatura ambiente las muestras. La absorbancia de las muestras se midió a 765 nm usando el espectrofotómetro Spectrophotometer Jenway 6320D, USA.

Para la determinación de fenoles totales, se hizo una curva de calibración (0,002 a 0,017 mg de ácido gálico (AG) por ml de solución, con una correlación de 99,55\%). Los valores fueron expresados en mg de ácido gálico /100 g de muestra b.s

\subsection{Determinación de la capacidad antioxidante (\% DPPH)}

La capacidad antioxidante expresada como porcentaje de inhibición del radical DPPH 2,2-diphenyl-1-picrylhydrazyl, se determina siguiendo la metodología 
de [23]. Se pesó $0,1 \mathrm{~g}$ de muestra en un tubo de ensayo forrado con papel aluminio y se adicionaron $10 \mathrm{ml}$ de metanol. Se agitó en una plancha de agitación a $150 \mathrm{rpm}$ durante tres minutos y posteriormente se sonificó la mezcla durante $20 \mathrm{~min}$ a $25^{\circ} \mathrm{C}$.

Pasado este tiempo, se filtró el extracto y se tomó $1 \mathrm{ml}$ de este para mezclarlo con 2 $\mathrm{ml}$ del reactivo DPPH $0,1 \mathrm{mM}$ recién preparado. Se elaboró un control con $1 \mathrm{ml}$ de metanol y $2 \mathrm{ml}$ de $\mathrm{DPPH}$ recién preparado. Se dejaron en reposo durante 1 $\mathrm{h}$ en la oscuridad la muestra y el control.

Luego se midió la absorbancia de la muestra y el control usando el espectrofotómetro Spectrophotometer Jenway 6320D, USA a una longitud de onda de $517 \mathrm{~nm}$.

El porcentaje de actividad antioxidante en términos de inhibición del radical DPPH se determinó mediante (4):

$$
\operatorname{DPPH}(\%)=\frac{\text { A517control }- \text { A517muestra }}{\text { A517Control }}
$$

Donde A517= lectura de la absorbancia a una longitud de onda de $517 \mathrm{~nm}$.

\subsection{Análisis estadístico}

La comparación de medias de los tratamientos fresco y deshidratado y la diferencia entre ellos se determinó mediante una prueba T-Student, y el efecto de la inclusión del epicarpio de mandarina en el pan se estableció por medio de un diseño experimental aleatorizado simple con cuatro tratamientos (control, $2 \%, 3 \% \mathrm{y}$ $4 \%)$ y una prueba Tukey para observar las diferencias entre los tratamientos. El tratamiento de datos es evaluado en el paquete estadístico minitab $V 17$ con un nivel de confianza del $95 \%$. La experimentación de todos los tratamientos se hizo por triplicado y se llevó a cabo en el laboratorio de Frutas y Hortalizas de la Universidad Nacional de Colombia sede Palmira.

\section{RESULTADOS Y DISCUSIÓN}

\subsection{Propiedades fisicoquímicas en la harina de epicarpio de mandarina}

En la Tabla 1 se presentan los valores medios evaluados de los parámetros fisicoquímicos: color superficial CIEL*a*b*, fenoles totales y capacidad antioxidante (\% DPPH), evaluada en el epicarpio de mandarina en fresco y deshidratado. El análisis estadístico evidencia un efecto significativo en las variables de estudio después del proceso de deshidratación, excepto en la variable de color $h^{\circ}$.

Los valores de porcentaje materia seca (\% materia seca), aw, $\mathrm{pH}, \mathrm{L}^{*}, \mathrm{y} \mathrm{C}^{*}$ del material en fresco superan significativamente los valores obtenidos en el material deshidratado, por el contrario, la acidez (\% acidez), los fenoles totales y la actividad antioxidante son significativamente mayores en el material deshidratado (Tabla 1).

El epicarpio de mandarina es deshidratado hasta alcanzar un porcentaje de materia seca de $91,63 \pm 2,52 \%$ y una

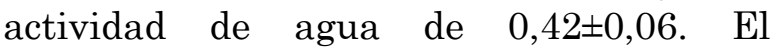
decrecimiento del agua disponible permite prolongar la vida útil de la misma, disminuyendo la proliferación de bacterias, hongos, mohos y reacciones enzimáticas de deterioro.

El epicarpio de mandarina en fresco presenta un $\mathrm{pH}$ de $5,19 \pm 0,10$ y un

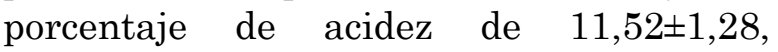
mientras que el epicarpio de mandarina

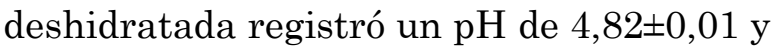
un porcentaje de acidez de 15,79 $\pm 2,41$.

Estos cambios en las muestras evaluadas después del tratamiento térmico pueden estar asociados a los procesos de lixiviación y oxidación de los compuestos orgánicos cuando la matriz biológica es afectada por la temperatura [24]. 
Potencial agroindustrial del epicarpio de mandarina como alternativa de colorante natural en pan

Tabla 1. Parámetros fisicoquímicos del epicarpio de mandarina en fresco y deshidratado Fuente: elaboración propia.

\begin{tabular}{lccc}
\hline Muestra $^{1}$ & Fresco & Deshidratado & T-Studet \\
\hline \% Materia Seca & $16,16 \pm 1,58$ & $91,63 \pm 2,52$ & $* * *$ \\
aw & $0,995 \pm 0,00$ & $0,42 \pm 0,06$ & $* * *$ \\
pH & $5,19 \pm 0,10$ & $4,82 \pm 0,01$ & $* *$ \\
\% acidez ${ }^{2}$ & $11,52 \pm 1,28$ & $15,79 \pm 2,41$ & $*$ \\
Fenoles totales ${ }^{3}$ & $19,81 \pm 0,60$ & $27,07 \pm 2,68$ & $* *$ \\
\% DPPH & $53,27 \pm 2,80$ & $88,79 \pm 0,93$ & $* * *$ \\
L $^{*}$ & $49,97 \pm 0,68$ & $39,74 \pm 0,05$ & $* * *$ \\
C $^{*}$ & $43,82 \pm 1,26$ & $25,08 \pm 0,13$ & $* * *$ \\
h $^{\text { degree }}$ & $81,99 \pm 4,55$ & $86,38 \pm 0,05$ & $\mathrm{NS}$ \\
\hline
\end{tabular}

1Valores promedios \pm DS (n=3)

${ }^{2}$ Valores expresados en equivalentes de ácido cítrico/ $100 \mathrm{~g}$ b.s

${ }^{3}$ Valores expresados en mg de ácido gálico /100g de muestra b.s

$\left({ }^{*}\right)$ : significativo $\mathrm{p}<0,05 ;\left({ }^{* *}\right)$ : significativo $\mathrm{p}<0,01 ;\left({ }^{* * *}\right)$ : significativo $\mathrm{p}<0,001 ;$ para dos muestras

NS: no hay diferencias significativas

El epicarpio de mandarina presenta bajos niveles de compuestos fenólicos totales; $19,81 \pm 0,60$ y $27,07 \pm 2,68 \mathrm{mg}$ de ácido gálico/100 g de muestra, en fresco y deshidratado respectivamente. De acuerdo con la clasificación propuesta por [25], la mandarina se clasifica como una fruta de bajo contenido de compuestos fenólicos, ya que el epicarpio presenta valores inferiores a $500 \mathrm{mg}$ de ácido gálico/100 g de muestra.

Esto concuerda con los resultados obtenidos por [26], que clasifica las variedades de mandarina clementina $\mathrm{y}$ mandarín Fairchild, Dancy, Beleza, Suhugan y Fizu como una fruta de bajo contenido de compuestos fenólicos, ya que estas presentan concentraciones menores a $75 \mathrm{mg}$ de ácido gálico/100 g de muestra. El aumento significativo en la cantidad de contenido de compuestos fenólicos en la muestra deshidratada puede ser causado por la aceleración de las reacciones de escisión de sustancias fenólicas reunidas en las células por el calentamiento [27].

Los compuestos fenólicos son sustancias que poseen un anillo aromático y un anillo de benceno con uno o más grupos de hidróxido, que incluyen derivados funcionales (como ésteres, ésteres metílicos, glucósidos, etc.) [28] que pueden actuar como agentes protectores contra la acción de la radiación UV, patógenos y depredadores en frutas y verduras [4], además, estos compuestos desactivan las moléculas de pro-carcinógenos y propician el mantenimiento de la reparación del ADN, la supresión de la formación de Nnitrosamina y el cambio del metabolismo del estrógeno, entre otros [8].

El 1,1-difenil-2-picrilhidrazilo (radical DPPH) es un radical libre estable centrado en nitrógeno, al aceptar un radical de hidrógeno o un electrón, el DPPH se convierte en una molécula diamagnética estable que desempeña el papel de un antioxidante [11]. El porcentaje de actividad de captación de DPPH en la muestra deshidratada $(88,79 \pm 0,93 \%)$ fue significativamente mayor al de la muestra en fresco $(53,27 \pm 2,80 \%)$. Este resultado concuerda con lo encontrado por [29], en cuyo trabajo se observó un aumento del porcentaje de actividad de eliminación de DPPH del $48 \%$ en flavedo de pomelo, después del tratamiento de secado en horno a $50{ }^{\circ} \mathrm{C}$, el autor obtuvo valores de $\%$ 
DPPH de $45,8 \%$ y $87,92 \%$ en fresco y deshidratado respectivamente.

La capacidad antioxidante de los extractos está influenciada por su composición química y actúa como agente protector reduciendo el daño oxidativo causado en el cuerpo, inhibiendo la peroxidación de los lípidos y retardando el progreso de muchas enfermedades [11].

Según [1], la capacidad antioxidante de los cítricos está asociada a compuestos como las vitaminas; entre estos se encuentran la vitamina $\mathrm{A}$, que puede reaccionar con los radicales libres, especialmente con el oxígeno singlete $\left({ }^{1} \mathrm{O}_{2}\right)$ $\mathrm{y}$ los radicales peroxilo; la vitamina $\mathrm{C}$, que puede eliminar eficazmente una variedad de especies reactivas de oxígeno (ROS) y desprender ácido semideshidroascórbico, eliminando ${ }^{1} \mathrm{O}_{2}$ y reduciendo los radicales de azufre; la vitamina $\mathrm{E}$, que puede proteger las membranas celulares contra el daño por la peroxidación de lípidos; minerales como el $\mathrm{Mn}, \mathrm{Fe}, \mathrm{Cu}, \mathrm{Zn}$ y Se; compuestos fenólicos como los flavonoides, que pueden eliminar especies reactivas de oxígeno y disminuir la formación de peróxido in vivo; ácidos fenólicos, a través de la deshidrogenación de los grupos hidroxilo; el efecto de la ortosustitución en un anillo de benceno y las curcuminas debido a sus grupos hidroxilo-fenólicos; y compuestos terpenoides como los limonoides, la pectina a través de la mejora de las enzimas antioxidantes endógenas y la eliminación de radicales libres y los carotenoides debido a la eliminación de radicales libres y ${ }^{1} \mathrm{O}_{2}$.

La reducción de los valores de color superficial $\mathrm{L}^{*}$ y $\mathrm{C}^{*}$ en las muestras deshidratadas concuerdan con los resultados obtenidos por [30] y [31] al deshidratar los subproductos generados en el procesamiento de frutos cítricos.

La reducción de estos parámetros de color durante el proceso de deshidratación pueden ser consecuencia de la isomeración de los carotenoides, polimerización y degradación de flavonoides o de las reacciones de pardeamiento no enzimático, tales como condensación de hexosas y componentes de aminoácidos, y la oxidación del ácido ascórbico, tal como lo expresó previamente [24].

\subsection{Concentración de carotenoides en la harina de epicarpio de mandarina}

Los carotenoides son tetraterenoides responsables principalmente del color rojo, naranja o amarillo de las frutas y son importantes precursores de la vitamina $\mathrm{A}$ [9]. También poseen varias propiedades funcionales, como el estímulo de la actividad cardiovascular, capacidad antioxidante y anticancerígeno [10].

En la Tabla 2 se presentan los valores de la concentración del contenido de carotenoides evaluados en el epicarpio de la mandarina en fresco y deshidratado. El análisis estadístico revela una diferencia significativa entre las muestras evaluadas.

Tabla 2. Contenido de carotenoides presentes en el epicarpio de mandarina en fresco y deshidratado Fuente: elaboración propia.

\begin{tabular}{lccc}
\hline Muestra $^{1}$ & Fresco & Deshidratado & T-Student \\
\hline B- caroteno & $20,81 \pm 3,58$ & $116,52 \pm 0,45$ & $* * *$ \\
a- caroteno & $22,16 \pm 4,59$ & $112,15 \pm 3,99$ & $* * *$ \\
B-criptoxantina & $21,17 \pm 3,24$ & $119,06 \pm 0,11$ & $* * *$ \\
zeaxantina & $21,00 \pm 3,21$ & $118,10 \pm 0,11$ & $* * *$ \\
licopeno & $16,58 \pm 0,62$ & $67,76 \pm 2,74$ & $* * *$ \\
\hline
\end{tabular}

1Valores promedios \pm DS ( $\mathrm{n}=3)$; los valores son expresados en $\mathrm{mg} / 100 \mathrm{~g}$ de muestra seca

$(* * *)$ : significativo $\mathrm{p}<0,001$, para dos muestras 
La retención del contenido de carotenoides obtenidos del epicarpio de mandarina en la muestra deshidratada fue significativamente mayor al material en fresco. Este aumento en la concentración de carotenoides se le atribuye al tratamiento térmico al que fue sometido el material en fresco durante el proceso de deshidratación [23], ya que este aumenta la biodisponibilidad de los carotenoides, al destruir la integridad de la pared celular y las membranas de los orgánulos en los que se encuentran retenidos. Además, el calor desnaturaliza los complejos proteínacarotenoides, favoreciendo su liberación de la matriz alimentaria [32], [33].

Tratamientos térmicos como el escaldado están asociados con la inhibición de la enzima lipoxigenasa (LOX), la cual está relacionada con la destrucción o blanqueado de pigmentos carotenoides [34].

Se obtiene una concentración significativamente mayor de los carotenoides $\alpha$ - caroteno y B-criptoxantina en el material en fresco, mientras que en el material deshidratado se obtienen concentraciones mayores en los carotenoides B-criptoxantina y zeaxantina.

El carotenoide B-criptoxantina es el responsable de la coloración naranja del epicarpio de mandarina [35], razón por la cual es uno de los carotenoides predominantes en las dos muestras evaluadas.

El licopeno fue el pigmento que presentó menor concentración tanto en fresco como deshidratado, se presentan concentraciones de $16,58 \pm 0,62 \mathrm{mg} / 100 \mathrm{~g}$ de muestra seca y $67,76 \pm 2,74 \mathrm{mg} / 100 \mathrm{~g}$ de muestra seca respectivamente.

Este carotenoide es considerado el pigmento con mayor capacidad para eliminar el oxígeno singlete. Los estudios han demostrado que el licopeno protege las moléculas lipídicas, las lipoproteínas de baja densidad, las proteínas y el ADN contra los ataques de radicales libres, desempeñando un papel esencial en la protección contra enfermedades [9].

La concentración obtenida del epicarpio de mandarina deshidratada de Bcaroteno y licopeno fue muy elevada comparada con los reportados por [36] en subproductos deshidratados derivados del procesamiento industrial de naranja y maracuyá; el autor reporta concentraciones de B-caroteno $39,14 \mu \mathrm{g} / \mathrm{g}$ y licopeno $18,51 \mu \mathrm{g} / \mathrm{g}$ para naranja y $\mathrm{B}$ - caroteno 56,07 $\mu \mathrm{g} / \mathrm{g}$ y licopeno $28,57 \mu \mathrm{g} / \mathrm{g}$ para maracuyá.

La concentración obtenida Bcriptoxantina en el epicarpio de mandarina fresca fue mayor que la reportada por [37].

$\mathrm{El}$ autor reporta valores de $13,4 \mu \mathrm{g} / \mathrm{g}$ en mandarina clementina, $11,4 \mu \mathrm{g} / \mathrm{g}$ en naranja china, $11,3 \mu \mathrm{g} / \mathrm{g}$ en pomelo $\mathrm{y}$ $19,9 \mu \mathrm{g} / \mathrm{g}$ en limón. Las variaciones de la concentración de carotenoides pueden atribuirse a: las prácticas agrícolas, exposición al sol, el área de producción, la etapa de maduración, el manejo pos cosecha y la metodología utilizada para el análisis [38].

\subsection{Valoración de la harina de epicarpio de mandarina como colorante natural en el pan}

Las propiedades fisicoquímicas del pan formulado con las diferentes concentraciones de harina de epicarpio de mandarina se representan en la Tabla 3.

El análisis de Anova revela que existen diferencias significativas entre el control y las formulaciones con el subproducto de la mandarina, excepto en el parámetro de color $h^{\circ}$ (Tabla 3). En el caso de los carotenoides, se observa un aumento significativo de estos pigmentos en el pan al incrementar la harina de epicarpio de mandarina (Tabla 3). [13], [18] y [39]-[41] concuerdan con nuestros resultados al utilizar subproductos de frutas en la formulación de productos horneados. 
Potencial agroindustrial del epicarpio de mandarina como alternativa de colorante natural en pan

Tabla 3. Concentración de carotenoides y color superficial con diferentes concentraciones de epicarpio de mandarina. Fuente: elaboración propia.

\begin{tabular}{|c|c|c|c|c|c|}
\hline \multirow[b]{2}{*}{ Variables $^{1}$} & \multicolumn{4}{|c|}{ Formulaciones } & \multirow{2}{*}{ Anova } \\
\hline & Control & $2 \%$ & $3 \%$ & $4 \%$ & \\
\hline B- caroteno ${ }^{2}$ & $2,23 \pm 1,51 \mathrm{~d}$ & $5,84 \pm 1,95 c$ & $8,96 \pm 1,22 b$ & $10,46 \pm 1,56 \mathrm{a}$ & $* * *$ \\
\hline a- caroteno ${ }^{2}$ & $1,64 \pm 1,52 \mathrm{~d}$ & $4,77 \pm 1,91 \mathrm{c}$ & $8,19 \pm 1,12 b$ & $14,10 \pm 1,98 \mathrm{a}$ & $* * *$ \\
\hline B-criptoxantina ${ }^{2}$ & $1,28 \pm 1,53 \mathrm{c}$ & $5,65 \pm 1,98 b$ & $6,91 \pm 1,63 b$ & $11,30 \pm 1,96 \mathrm{a}$ & $* * *$ \\
\hline Zeaxantina $^{2}$ & $1,26 \pm 1,54 \mathrm{c}$ & $4,58 \pm 2,64 \mathrm{~b}$ & $4,78 \pm 2,02 \mathrm{~b}$ & $10,27 \pm 2,04 \mathrm{a}$ & $* * *$ \\
\hline Licopeno $^{2}$ & $0,88 \pm 1,55 \mathrm{c}$ & $3,21 \pm 1,85 b$ & $3,35 \pm 1,42 \mathrm{~b}$ & $7,20 \pm 1,43 \mathrm{a}$ & $* * *$ \\
\hline $\mathrm{L}^{*}$ & $\begin{array}{l}37,19 \pm 2,71 \\
\text { b }\end{array}$ & $74,06 \pm 1,98 \mathrm{a}$ & $73,17 \pm 2,04 \mathrm{a}$ & $73,25 \pm 2,18 \mathrm{a}$ & $* * *$ \\
\hline $\mathrm{C}^{*}$ & $\begin{array}{l}20,42 \pm 1,55 \\
\text { b }\end{array}$ & $32,21 \pm 0,81 \mathrm{a}$ & $35,36 \pm 1,44 \mathrm{a}$ & $38,51 \pm 1,55 \mathrm{a}$ & $* *$ \\
\hline$h^{o}$ & $\begin{array}{l}92,95 \pm 1,09 \\
\text { a }\end{array}$ & $93,16 \pm 0,82 \mathrm{a}$ & $92,71 \pm 0,53 \mathrm{a}$ & $92,44 \pm 0,56 \mathrm{a}$ & NS \\
\hline
\end{tabular}

${ }^{1}$ Valores promedios $\pm \operatorname{DS}(\mathrm{n}=3)$; Las diferentes letras en cada fila indican una diferencia significativa $(* *) \mathrm{p}<0,01$, $(* * *) \mathrm{p}<0,001$ entre cada formulación

${ }^{2}$ los valores son expresados en $\mathrm{mg} / 100 \mathrm{~g}$ de muestra seca

Por otra parte, al comparar las concentraciones presentes de estos pigmentos en el epicarpio deshidratado y el pan, se observa una reducción de carotenoides (Tabla 2, y 3). Autores como [42] afirman que estas pérdidas pueden estar asociadas a los procesos de amasado y horneado, a la incorporación de agua, y al oxígeno en la masa, que permite la activación de la lipoxigenasa (LOX), responsable de la oxidación enzimática de los ácidos grasos poliinsaturados, que a su vez desencadena la oxidación de los carotenoides, que, a su vez, durante temperaturas de cocción pueden iniciar procesos de oxidación e isomerización en los carotenoides. Las pérdidas de carotenoides durante la producción de alimentos horneados han sido reportadas previamente por [13], [18], [39]-[41].

En el pan, los atributos de color $\left(\mathrm{L}^{*}\right)$ y cromaticidad $\quad\left(\mathrm{C}^{*}\right) \quad$ aumentan significativamente al incrementar el epicarpio de mandarina en la formulación (Tabla 3). Los resultados de $\mathrm{L}^{*}$ y $\mathrm{C}^{*}$ no se ajustan a los registrados previamente por [13]. Por otra parte, el hecho de que no existan diferencias significativas del tono $\left(h^{\circ}\right)$ entre el control y las formulaciones propuestas en el presente estudio indica que los productos evaluados (control y formulaciones con subproducto) generan una percepción de color amarillo al ser revisados con las coordenadas CIEL*a*b*, evidenciando el potencial agroindustrial de este residuo como una alternativa de aditivo colorante en la elaboración del pan.

La explicación de esta propiedad en el pan elaborado con epicarpio de mandarina está asociada a la presencia de carotenoides en este subproducto, tal como se ha registrado previamente en la Tabla 2 .

\section{CONCLUSIONES}

En el presente estudio se puede concluir que el epicarpio deshidratado de mandarina de variedad Oneco es una importante fuente de carotenoides y compuestos antioxidantes que podrían ser utilizados como aditivos naturales en la industria alimentaria, farmacéutica y cosmética. Por otra parte, el proceso de deshidratación reduce significativamente la intensidad del color amarillo en el epicarpio de mandarina; sin embargo, este subproducto podría ser una importante alternativa de aditivo colorante natural en la elaboración de pan.

Se recomienda desarrollar estudios sobre estabilidad, calidad y análisis sensorial del pan formulado con la harina 
de epicapio de mandarina durante el almacenamiento.

\section{AGRADECIMIENTOS}

Los autores manifiestan su agradecimiento a la Universidad Nacional de Colombia, Sede Palmira, a través del Proyecto Hermes 42088 y al grupo de investigación Procesos Agroindustriales (GIPA).

\section{CONFLICTOS DE INTERÉS}

Ningún autor tiene conflicto alguno que declarar.

\section{REFERENCIAS}

[1] Z. Zou, W. Xi, Y. Hu, C. Nie y Z. Zhou, "Antioxidant activity of Citrus fruits", Food Chem., vol. 196, pp. 885-896, Abr. 2016. https://doi.org/10.1016/j.foodchem.2015.09.072

[2] M. F. Martínez, "Caracterización molecular de genotipos de mandarinas citrus spp. mediante marcadores ram's (microsatélites amplificados al azar) y microsatélites" (tesis de Marestría) Universidad Nacional de Colombia, Palmira, 2013. Disponible en: URL

[3] Taxonomy Browser, “Trópicos”, 2018. Disponible en: URL

[4] H. R. de Moraes Barro, T. A. Pinto de Castro Ferreira y M. I. Genovese, "Antioxidant capacity and mineral content of pulp and peel from commercial cultivars of citrus from Brazil", Food Chem., vol. 134, no. 4, pp. 1892 1898,

2012. https://doi.org/10.1016/j.foodchem.2012.03.090

[5] J. Contreras-Calderón, L. Calderón-Jaimes, E. Guerra-Hernández y B. García-Villanova, "Antioxidant capacity, phenolic content and vitamin $\mathrm{C}$ in pulp, peel and seed from 24 exotic fruits from Colombia", Food Res. Int., vol. 44, no.7, pp. 2047-2053, ago. 2011. https://doi.org/10.1016/j.foodres.2010.11.003

[6] N. M'hiri, I. Ioannou, M. Ghoul y N. Mihoubi Boudhrioua, "Phytochemical characteristics of citrus peel and effect of conventional and nonconventional processing on phenolic compounds: A review", Food Rev. Int., vol. 33, no.6, pp. 587-619, Jun. 2017. https://doi.org/10.1080/87559129.2016.119648 $\underline{9}$

M. Sanz-Puig, P. Moreno, M. C. Pina-Pérez, D. Rodrigo y A. Martínez, "Combined effect of High Hydrostatic Pressure (HHP) and antimicrobial from agro-industrial byproducts against S. Typhimurium", $L W T$, vol. 77, pp. 126-133, abr. 2017. https://doi.org/10.1016/j.lwt.2016.11.031

[8] S. Rafiq, R. Kaul, S. A. Sofi, N. Bashir, F. Nazir y G. Ahmad Nayik, "Citrus peel as a source of functional ingredient: A review", $J$. Saudi Soc. Agric. Sci., vol. 17, no.4, pp. 351358, $\quad$ oct. 2018. https://doi.org/10.1016/j.jssas.2016.07.006

[9] L. M. R. da Silva et al., "Quantification of bioactive compounds in pulps and by-products of tropical fruits from Brazil", Food Chem., vol. 143, pp. 398-404, ene. 2014.

https://doi.org/10.1016/j.foodchem.2013.08.001

[10] K. M. Yoo y B. K. Moon, "Comparative carotenoid compositions during maturation and their antioxidative capacities of three citrus varieties", Food Chem., vol. 196, pp. 544-549, abr. 2016. https://doi.org/10.1016/j.foodchem.2015.09.079

[11] B. B. Mathew, D. Shajie, N. Wadhwa, N. B. Krishna Murthy, T. P. Krishna Murthy y M. Rashmi, "Comparative antioxidant efficacy of Citrus limonum pulp and peel. An in vitro study", Drug Invent. Today, vol. 5, no.4, pp. 296-301, dic. 2013. https://doi.org/10.1016/j.dit.2013.07.003

[12] V. Goulas y G. A. Manganaris, "Exploring the phytochemical content and the antioxidant potential of Citrus fruits grown in Cyprus", Food Chem., vol. 131, no.1, pp. 39-47, Mar. 2012.

https://doi.org/10.1016/j.foodchem.2011.08.007

[13] L. E. Ordóñez-Santos, J. Martínez-Girón y A. M. Figueroa-Molano, "Effect of the addition of peach palm (Bactris gasipaes) peel flour on the color and sensory properties of cakes", Food Science and Technology, vol. 37 , no.3, pp. 418-424, Jul. 2017.

https://doi.org/10.19084/rca16008

[14] G. D. Singh, C. S. Riar, C. Saini, A. S. Bawa, D. S. Sogi y D. C. Saxena, "Indian water chestnut flour- method optimization for preparation, its physicochemical, morphological, pasting properties and its potential in cookies preparation", LWT-Food Sci. Technol., vol. 44, no.3, pp. 665-672, abr. 2011. https://doi.org/10.1016/j.lwt.2010.09.015

[15] L. C. R. dos Reis, E. M. P. Facco, M. Salvador, S. H. Flôres y A. de O. Rios, "Characterization of Orange Passion Fruit Peel Flour and Its Use as an Ingredient in Bakery Products", $J$. Culin. Sci. Technol., pp. 1-17. dic. 2018. https://doi.org/10.1080/15428052.2018.156410 $\underline{3}$ 
Potencial agroindustrial del epicarpio de mandarina como alternativa de colorante natural en pan

[16] H. S. Ali, A. Farouk Mansour, M. M. Kamil y A. M. S. Hussein, "Formulation of Nutraceutical Biscuits Based on Dried Spent Coffee Grounds", Int. J. Pharmacol., vol. 14, no. 4, pp. 584-594, Abr. 2018 . https://doi.org/10.3923/ijp.2018.584.594

[17] D. Potosi Calvache, P. Vanegas Mahecha y H. A. Martínez, Correa, "Convective drying of squash (Cucurbita moschata): Influence of temperature and air velocity on effective moisture diffusivity, carotenoid content and total phenols", Dyna, vol. 84, no.202, pp. 112 119, jul. 2017.

https://doi.org/10.15446/dyna.v84n202.63904

[18] L. E. Ordóñez-Santos, J. Martínez-Girón y A. M. Figueroa-Molano, "Effect of the addition of peach palm (Bactris gasipaes) peel flour on the color and sensory properties of wheat bread", Rev. Ciências Agrárias, vol. 39, no.3, pp. 456-462, ene. 2016.

https://dx.doi.org/10.19084/RCA16008

[19] AOAC, Official Methods Of Analysis of AOAC International, 17th Edn. Gaithersburg (Maryland): Agricultural Chemicals, contaminants, drugs AOAC, 1997. Disponible en: URL

[20] Icontec. Instituto Colombiano de Normas Técnicas y Certificación. Norma Técnica Colombiana. NTC 4592, "Productos de frutas y verduras.determinación del pH”, p. 4, 1999. Disponible en: URL

[21] AOC International,"Official Methods of Analysis method 936.07 Ash of Flour”, vol. 5. Gaithersburg, 1999. Disponible en: URL

[22] L. E. Ordóñez-Santos, J. Martínez-Girón y M. E. Arias-Jaramillo, "Effect of ultrasound treatment on visual color, vitamin $\mathrm{C}$, total phenols $y$ carotenoids content in Cape gooseberry juice", Food Chem., vol. 233, pp. 96-100, oct. 2017. https://doi.org/10.1016/j.foodchem.2017.04.114

[23] J. Martínez-Girón, X. Rodríguez-Rodríguez, L. X. Pinzón-Zárate y L. Ordóñez-Santos, "Caracterización fisicoquímica de harina de residuos del fruto de chontaduro (Bactris gasipaes Kunth, Arecaceae) obtenida por secado convectivo", Corpoica Cienc Tecnol Agropecu., vol. 18, no.3, pp. 599-613, ago. 2017.

https://doi.org/10.21930/rcta.vol18_num3_art: $\underline{747}$

[24] H. G. Cuastumal Canacuan, B. L. Valencia Murillo y L. E. Ordoñez Santos, "Efectos de los tratamientos térmicos en la concentración de vitamina $\mathrm{C}$ y color superficial en tres frutas tropicales", Rev. Lasallista Investig., vol. 13, no.1, pp. 85-93, jun. 2016. https://doi.org/10.22507/rli.v13n1a7

[25] C. Vasco, J. Ruales y A. Kamal-Eldin, "Total phenolic compounds and antioxidant capacities of major fruits from Ecuador", Food Chem., vol. 111, no. 4, pp. 816-823, dic. 2008. https://doi.org/10.1016/j.foodchem.2008.04.054

[26] D. Ramful, E. Tarnus, O. I. Aruoma, E. Bourdon y T. Bahorun, "Polyphenol composition, vitamin $\mathrm{C}$ content and antioxidant capacity of Mauritian citrus fruit pulps", Food Res. Int., vol. 44, no. 7, pp. 20882099, Aug, 2011.

https://doi.org/10.1016/j.foodres.2011.03.056

[27] Y. Tekgül y T. Baysal, "Comparative evaluation of quality properties and volatile profiles of lemon peels subjected to different drying techniques", J. Food Process Eng., vol. 41, no. 8, pp. 1-9, oct. 2018. https://doi.org/10.1111/jfpe.12902

[28] E. Roselló-Soto et al., "Clean recovery of antioxidant compounds from plant foods, byproducts and algae assisted by ultrasounds processing. Modeling approaches to optimize processing conditions", Trends Food Sci. Technol., vol. 42, no. 2, pp. 134-149, abr. 2015. https://doi.org/10.1016/j.tifs.2015.01.002

[29] N. F. A. Rahman, R. Shamsudin, A. Ismail, N. N. A. Karim Shah y J. Varith, "Effects of drying methods on total phenolic contents and antioxidant capacity of the pomelo (Citrus grandis (L.) Osbeck) peels", Innov. Food Sci. Emerg. Technol., vol. 50, pp. 217225, $\quad$ dic. 2018. https://doi.org/10.1016/j.ifset.2018.01.009

[30] X. A. Ruiz-Armenta et al., "Effect of extrusion on the carotenoid content, physical and sensory properties of snacks added with bagasse of naranjita fruit: optimization process", CyTA-J. Food, vol. 16, no.1, pp. 172180, sep. 2017. https://doi.org/10.1080/19476337.2017.136871 7

[31] M. T. Pacheco, F. J. Moreno y M. Villamiel, "Chemical and physicochemical characterization of orange by-products derived from industry", Sci. Food Agric., vol. 99, no. 2, pp. 868-876, Jan. 2019. https://doi.org/10.1002/jsfa.9257

[32] A. Cilla, L. Bosch, R. Barberá y A. Alegría, "Effect of processing on the bioaccessibility of bioactive compounds. A review focusing on carotenoids, minerals, ascorbic acid, tocopherols and polyphenols", J. Food Compos. Anal., vol. 68, pp. 3-15, may. 2018. https://doi.org/10.1016/j.jfca.2017.01.009

[33] L. Cárcamo-Medina, M. Elezar-Turcios y L. Ordóñez-Santos, "Cambios en los compuestos bioactivos del jugo de uchuva (Physalis peruviana L) pasteurizado", TecnoLógicas, vol. 22 , no.45, pp. 145-153, may. 2019. https://doi.org/10.22430/22565337.1279

[34] S. Hayward, T. Cilliers y P. Swart, "Lipoxygenases: From Isolation to 
Application", Compr. Rev. Food Sci. Food Saf., vol. 16, n. ${ }^{\circ} 1$, pp. 199-211, ene. 2017. https://doi.org/10.1111/1541-4337.12239

[35] A. Conesa et al., "Changes in the content of chlorophylls and carotenoids in the rind of Fino 49 lemons during maturation and their relationship with parameters from the Cielab color space", Sci. Hortic. (Amsterdam), vol. 243, no. 3, pp. 252-260, Jan. 2019.

https://doi.org/10.1016/j.scienta.2018.08.030

[36] S. N. Casarotti, T. F. Borgonovi, C. L. F. M. Batista y A. L. B. Penna, "Guava, orange and passion fruit by-products: Characterization and its impacts on kinetics of acidification and properties of probiotic fermented products", $L W T$, vol. 98, pp. 69-76, Dec. 2018. https://doi.org/10.1016/j.lwt.2018.08.010

[37] A. Agócs, V. Nagy, Z. Szabó, L. Márk, R. Ohmacht y J. Deli, "Comparative study on the carotenoid composition of the peel and the pulp of different citrus species", Innov. Food Sci. Emerg. Technol., vol. 8, pp. 390-394, Sep. 2007.

https://doi.org/10.1016/j.ifset.2007.03.012

[38] E. Vieira da Silva Júnior, L. Lins de Melo, R. A. Batista de Medeiros, Z. M. Pimenta Barros y P. M. Azoubel, "Influence of ultrasound and vacuum assisted drying on papaya quality parameters", $L W T$, vol. 97, pp. 317-322, Nov. 2018. https://doi.org/10.1016/j.lwt.2018.07.017

[39] C. M. Ajila, M. Aalami, K. Leelavathi, y U. J. S. Rao, "Mango peel powder: A potential source of antioxidant and dietary fiber in macaroni preparations," Innov. Food Sci. Emerg. Technol., vol. 11, no. 1, pp. 219-224, Jan. 2010.

https://doi.org/10.1016/j.ifset.2009.10.004

[40] C. M. Ajila, K. Leelavathi y U. J. S. PrasadaRao, "Improvement of dietary fiber content and antioxidant properties in soft dough biscuits with the incorporation of mango peel powder", J. Cereal Sci., vol. 48, no. 2, pp. 319326, Sep. 2008.

https://doi.org/10.1016/j.jcs.2007.10.001

[41] M. H. Azizi, J. S. Andrade, J. S. Lemos, S. J. Souza, R. S. De Souza y Z. Hadian, "Quality of wheat bread incorporated with different levels of peach palm flour (Bactris gasipaes Kunth)", Trop. Agric. Food Sci., vol. 40, no. 1 1 pp. 13-22, 2012. Disponible en: URL
[42] A. Hidalgo, A. Brandolini y C. Pompei, "Carotenoids evolution during pasta, bread and water biscuit preparation from wheat flours", Food Chem., vol. 121, no. 3, pp. 746751, Aug. 2010.

https://doi.org/10.1016/j.jcs.2010.06.002

\section{CONTRIBUCIÓN DE LOS AUTORES}

D1 Investigador principal del proyecto de investigación Evaluación del potencial agroindustrial de los pigmentos carotenoides extraídos de piel de mandarina como colorante natural en productos de panificación, que permitió el desarrollo de la presente investigación; líder de la presente investigación, responsable del componente metodológico de las variables de respuesta, análisis estadístico y de la escritura del documento científico.

(D) 2 Estudiante de maestría del proyecto de investigación Evaluación del potencial agroindustrial de los pigmentos carotenoides extraídos de piel de mandarina como colorante natural en productos de panificación, que permitió el desarrollo de la presente investigación; responsable de la ejecución experimental y apoyo en la escritura del documento científico.

(D) 3 Coautor del proyecto de investigación Evaluación del potencial agroindustrial de los pigmentos carotenoides extraídos de piel de mandarina como colorante natural en productos de panificación, que permitió el desarrollo de la presente investigación; responsable del apoyo del componente metodológico, orientación en la preparación y adecuación de las muestras de estudio. 